Conclusions The annual incidence of paediatric ACL-reconstructions in Norway increased from 2005 to 2019. ACL injury imposes an increasing health burden on the young population which warrants further attention - especially regarding prevention.

\section{HIGH INCIDENCE OF ANTERIOR CRUCIATE LIGAMENT INJURIES WITHIN THE TWO FIRST MONTHS OF THE SEASON IN AMATEUR TEAM BALL SPORTS}

\begin{abstract}
${ }^{1,2}$ Caroline Mouton, ${ }^{2}$ Alli Gokeler, ${ }^{1,3}$ Anouk Urhausen, ${ }^{1,2}$ Christian Nührenbörger, 1,2,3 Romain Seil. 'Sports Clinic, Centre Hospitalier de Luxembourg - Clinique d'Eich, Luxembourg, Luxembourg; '2 Luxembourg Institute of Research in Orthopedics, Sports Medicine and Science, Luxembourg, Luxembourg; ${ }^{3}$ Sports Medicine Research Laboratory, Luxembourg Institute of Health, Luxembourg, Luxembourg
\end{abstract}

\subsection{6/bjsports-2021-IOC.369}

Background The incidence of anterior cruciate ligament (ACL) injuries is commonly reported as an annual rate. There is relatively little information about their seasonal aspects.

Objective The aim was to analyze the distribution of ACL injuries during the season in amateur football, handball and basketball. It was hypothesized that ACL injuries they were more common after the summer break in comparison to the rest of the season.

Design Retrospective case series

Setting Hospital-based registry

Patients Three hundred and seventy-one (282 males, 89 females) injuries were included according to the following criteria: ACL injury occurring in football $(n=258)$, handball $(n=56)$ or basketball $(n=57)$ and confirmed through clinical examination, MRI and arthroscopy where applicable, under 35 years of age and pre-injury participation in competitive sport. Interventions (or Assessment of Risk Factors) Gender; age; sport at injury; injury mechanism; previous or contralateral ACL injury.

Main Outcome Measurements The injury occurrence during the calendar year was divided into six periods of two months. Segment 1 (S1) represented the first two months after summer break (Football: September/October, handball/basketball: October/November).

Results The occurrence of ACL injuries differed significantly between segments $(p<0.01)$ and according to sports $(p<0.01)$. Almost one third occurred in S1 $(n=104 ; 28 \%)$. The distribution was similar in other segments (S2: 13\%, S3: 16\%, S4: 16\%; S5: 12\%, S6: 15\%). Fewer ACL injuries occurred in S2 in football (9\%) compared to basketball (23\%) $(\mathrm{p}<0.05)$. More ACL injuries occurred in S5 in football (17\%) compared to handball $(2 \%)$ and basketball $(2 \%)(\mathrm{p}<0.05)$.

Conclusions The majority of ACL injuries in amateur football, handball and basketball sports occurred immediately after the summer break in the first two months of the season. This indicates that amateur athletes should start ACL injury prevention programs before the start of the season to allow for gradual increases of load.

\section{PATIENTS WITH KNEE INJURY HAVE WORSE POSTURAL ORIENTATION THAN HEALTHY CONTROLS}

Jenny Nae, Eva Ageberg. Lund University, Lund, Sweden
Background Patients with knee injury appear to have worse postural orientation (i.e., alignment between body segments), at the knee, measured with gold standard three-dimensional motion analysis, compared with controls. However, there is a lack of studies on postural orientation for other joints than the knee, e.g., hip and trunk, as well as with a clinically applicable method.

Objective To investigate whether visual assessment of Postural Orientation Errors (POEs) differs between patients with anterior cruciate ligament reconstruction (ACLR) and healthy controls.

Design Cross-sectional study

Setting Clinical setting

Participants Inclusion criteria were: age 18-39 years, >16 weeks post-ACLR, and initiated jumping exercises. Fifty-three patients with ACLR (45\% women), mean age 26.7 (SD 6.5) years, and 30 controls (50\% women), mean age 28 (SD 7.9) years, were included.

Interventions Six POEs of the lower extremity and trunk, e.g., knee medial-to-foot position and femoral valgus, were visually assessed as good, fair, or poor, from video-recordings of 5 functional tasks. A score from 0 (good) to 100 (poor) was calculated for two subscales; activities of daily living (ADL) (single-leg squat, stair descending, forward lunge) and Sport (single-leg hop, side-hop), and a Total POE score (all 5 tasks). The injured leg was assessed in patients, and the right leg in controls.

Main Outcome Measurements POE scores

Results Patients with ACLR had significantly worse POE scores compared with controls, POE subscale ADL (ACLR: median 18.5 (quartiles 11-26), controls: 11 (3.7-14.8), $\mathrm{p}=0.0001)$, POE subscale Sport (ACLR: 26 (16.7-29.6), controls: 7.4 (3.7-15.7), $\mathrm{p}=0.0001$ ), and Total POE score (ACLR: 23.2 (14.3-25.9), controls: 8 (5.4-16), $\mathrm{p}=0.0001)$.

Conclusions Patients with ACLR appear to have worse postural orientation, measured with visual assessment, compared with controls. Visual assessment of POEs could be used to guide injury treatment aimed at improving postural orientation before return to sport, and potentially also to guide prevention training.

\section{DOES SOMATOSENSORY DYSFUNCTION EXIST AT RETURN TO PLAY FOLLOWING CONCUSSION IN ELITE ATHLETES}

${ }^{1,2}$ Theo Farley, ${ }^{1}$ Ed Owen, ' Louise Turner. ${ }^{1}$ The English Institute of Sports, London, UK; ${ }^{2}$ The Institute of Sport Exercise and Health, London, UK

\subsection{6/bjsports-2021-IOC.371}

Background A growing body of evidence suggests athletes may be over two times more likely to sustain a musculoskeletal (MSK) injury following concussion, highlighting significant clinical need to understand the pathophysiological changes in function and guide long term management of these patients. Reduced somatosensory function is considered one potential influencing factor for this phenomenon, whereby decreased postural control and bodily awareness leads to poor technique or abnormal movement strategies

Objective Using a measure of postural sway (Sway medical app, SMBA) and a balance assessment marker (Y-balance)this study aims to establish if somatosensory function is reduced at the point or return to play in a group of elite athletes. 